\title{
CRYOGENIC CHARACTERIZATION AND TESTING OF MAGNETICALLY-ACTUATED MICROSHUTTER ARRAYS FOR THE JAMES WEBB SPACE TELESCOPE
}

\author{
T.T. King", G. Kletetschka* ${ }^{\star}$, M.A. Jah", M.J. Li", M.D. Jhabvala*, L.L. Wang ${ }^{*}{ }^{*}$, M.A. Beamesderfer ${ }^{*}$, A.S. \\ Kutyrev $^{*}$, , R.F. Silverberg ${ }^{*}$, D. Rapchun ${ }^{*}{ }^{+}$, D.S. Schwinger ${ }^{*}$, G.M. Voellmer ${ }^{*}$, S.H. Moseley ${ }^{*}$, L.M. Sparr ${ }^{*}$ \\ *NASA Goddard Space Flight Center, Greenbelt, MD 20771, USA \\ †The Catholic University of America, Washington D.C. 20064, USA \\ ${ }^{\ddagger}$ Swales Aerospace Co., Greenbelt, MD 20705, USA \\ ${ }^{\S}$ Science Systems and Applications, Inc., Lanham, MD 20706, USA \\ ${ }^{+}$Global Science and Technology, Greenbelt, MD 20770, USA
}

\begin{abstract}
Two-dimensional MEMS microshutter arrays (MSA) have been fabricated at the NASA Goddard Space Flight Center (GSFC) for the James Webb Space Telescope (JWST) to enable cryogenic $(\sim 35 \mathrm{~K})$ spectrographic astronomy measurements in the nearinfrared region. Functioning as a focal plane object selection device, the MSA is a 2-D programmable aperture mask with fine resolution, high efficiency and high contrast. The MSA are closepacked silicon nitride shutters (cell size of 100 x $200 \mu \mathrm{m}$ ) patterned with a torsion flexure to allow opening to 90 degrees. A layer of magnetic material is deposited onto each shutter to permit magnetic actuation. Two electrodes are deposited, one onto each shutter and another onto the support structure side-wall, permitting electrostatic latching and 2-D addressing. New techniques were developed to test MSA under mission-similar conditions $(8 \mathrm{~K} \leq \mathrm{T}$ $<300 \mathrm{~K})$. The "magnetic rotisserie" has proven to be an excellent tool for rapid characterization of MSA. Tests conducted with the magnetic rotisserie method include accelerated cryogenic lifetesting of unpackaged $128 \times 64$ MSA and parallel measurement of the magneto-mechanical stiffness of shutters in "pathfinder" test samples containing multiple MSA designs. Lifetest results indicate a logarithmic failure rate out to $\sim 10^{6}$ shutter actuations. These results have increased our understanding of failure mechanisms and provide a means to predict the overall reliability of MSA devices.
\end{abstract}

\section{INTRODUCTION}

In the quest to reveal the origins of galaxies, clusters, and large-scale structures in the universe, scientists and engineers at the NASA GSFC have begun work on JWST scheduled to launch in 2011. To fulfill its primary mission of observing galaxies at the peak of the star-forming and merging era, JWST will carry the Near Infrared Spectrograph ${ }^{a}$ (NIRSpec) which will provide the required spectrographic coverage in the near-infrared wavelength region from 0.6 to $5 \mu \mathrm{m}$. To increase observation efficiency, simultaneous analysis of multiple astronomical bodies in the JWST/NIRSpec field of view will be enabled by a programmable aperture mask (PAM) that will select objects to optimally fill the focal plane without spectral overlap [1].

The science requirements on the PAM are that it must cover a large optical field of view in the focal plane $(7.7 \times 7.0 \mathrm{~cm}$ format area) with high resolution $(100 \times 200 \mu \mathrm{m}$ pixel dimensions), exhibit high on/off contrast ratio (> 2000), and a high fill factor ( $>$ $70 \%$ ). Furthermore, to minimize thermal emissions into NIRSpec and since the JWST second Lagrange point orbit will be

\footnotetext{
${ }^{\mathrm{a}}$ To be built by the European Space Agency.
}

inaccessible to space shuttle re-servicing missions, the PAM must operate in a cryogenic $(\sim 35 \mathrm{~K})$ vacuum environment reliably over a 10 year mission lifetime without repair after launch. Additionally, any device built to fly in space must, in general, have very tight constraints on mass, volume and power dissipation. In light of these mission requirements, microelectronic mechanical systems (MEMS) technologies are ideally suited for the design and development of the PAM.

\section{MICROSHUTTER ARRAY DESIGN}

A team at the NASA GSFC is developing a MEMS-based microshutter array (MSA) for application as the PAM on the JWST/NIRSpec $[\mathbf{2}, \mathbf{3}]$. The MSA will represent the first missioncritical MEMS device to be flown in space. The MSA design has been driven by the aforementioned science and mission requirements and will be verified through extensive characterization and testing under flight-similar conditions. The MSA flight concept consists of a $2 \times 2$ format mosaic of four $384 \times$ 175 arrays of close-packed shutters with a unit cell size of $100 \mathrm{x}$ $200 \mu \mathrm{m}$ placed in the JWST optical path at the focal plane. Individual shutters are designed with a torsion flexure hinge and are magnetically actuated by scanning a permanent tripole magnet across the MSA and electrostatically held open to allow the highcontrast transmission of light from specific celestial objects into NIRSpec. The selective nature of the MSA is achieved electrostatically via a three-voltage level, cross-point addressing scheme operating on orthogonally oriented column and row electrodes driven by external electronics $[4,5]$.

The challenges facing the MSA program are 1) the successful fabrication and packaging of the MSA, and 2) the development of a comprehensive test plan with MSA-specific test techniques to fully evaluate MSA parts under flight-similar conditions. This paper will highlight the progress achieved in these two areas and discuss recent results.

\section{MICROSHUTTER ARRAY FABRICATION}

The current MSA development focuses on refining fabrication and testing techniques on smaller 128 x 64 MSA prototypes before scaling up the design to flight-sized $384 \times 175$ MSA dice. The $128 \times 64$ MSA die are fabricated from silicon-oninsulator (SOI) wafers with a $0.5 \mu \mathrm{m}$ thick silicon nitride layer predeposited onto the oxide ${ }^{b}$. The processing on the front-side (the side for shutters) includes multi-layer metal deposition and patterning to create aluminum front-side electrodes, cobalt-iron

\footnotetext{
${ }^{\mathrm{b}}$ Obtained from MEMS Exchange, Reston, VA 20191.
} 
magnetic stripes ${ }^{c}$. A front side reactive ion etch (RIE) carves the shutters and torsion flexures out of the nitride membrane such that the front-side electrodes and magnetic stripes are made on each shutter blade. Each column of shutters is electrically contiguous through a single front-side strip electrode but electrically isolated from adjacent columns. An additional front-side metal deposition and patterning step is performed to build aluminum light shields around each shutter for blocking light leaks through the gap between the shutter and the silicon frame. The processing continues on the back-side (the side without shutters) with an anisotropic wafer-thinning back-etch, followed by a deep RIE back-etch to form a silicon frame support structure and to free the shutters from the silicon/oxide substrate. Additional back-side metal deposition, and patterning form back-side electrodes onto the silicon frame support structure and one of the side-walls. Each row of shutters is electrically contiguous through a single backside strip electrode but electrically isolated from adjacent rows [4, $5,6]$. A schematic representation of an individual shutter cell in cross-section and secondary electron micrographs of a fabricated 128 x 64 MSA die are displayed in Figures 1 and 2, respectively.

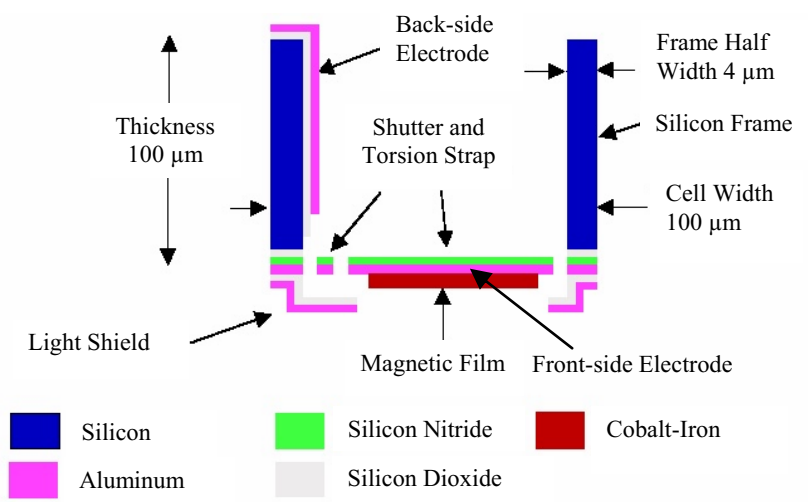

Figure 1. Schematic cross-section of a shutter cell.

Current packaging of 128 x 64 MSA dice involves bumpbonding the front-side electrode pads, and wire-bonding the backside electrode pads to gold traces on a $500 \mu \mathrm{m}$ thick silicon substrate. The silicon substrate is then wire-bonded to a printed circuit board $(\mathrm{PCB})^{\mathrm{d}}$ equipped with electrical pin connector interfaces to the drive electronics.

\section{MICROSHUTTER ARRAY TESTING}

A MSA test plan has been developed to evaluate $128 \times 64$ MSA parts that are produced to quickly refine the design and fabrication process to meet the PAM science and mission requirements. A host of MSA-specific test techniques and capabilities have been developed and are applied at various stages in the $128 \times 64$ MSA development. Some tests conducted on partially processed $128 \times 64$ MSA dice include (a) microscopic

c Cobalt-iron film deposited by the U.S. Naval Research Laboratory, Washington, DC 20375.

d Bonding done by the Johns Hopkins University Applied Physics Laboratory, Laurel, MD 20723.

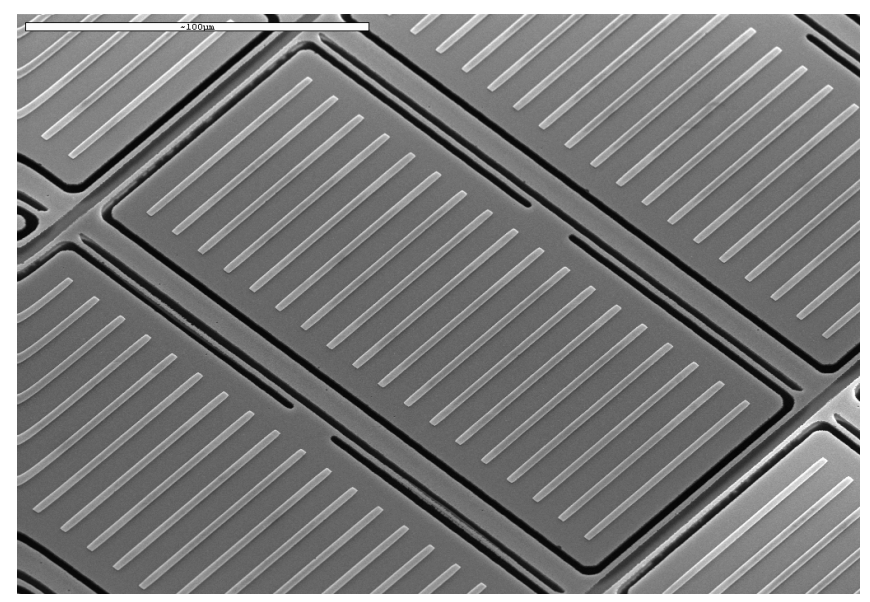

(a)

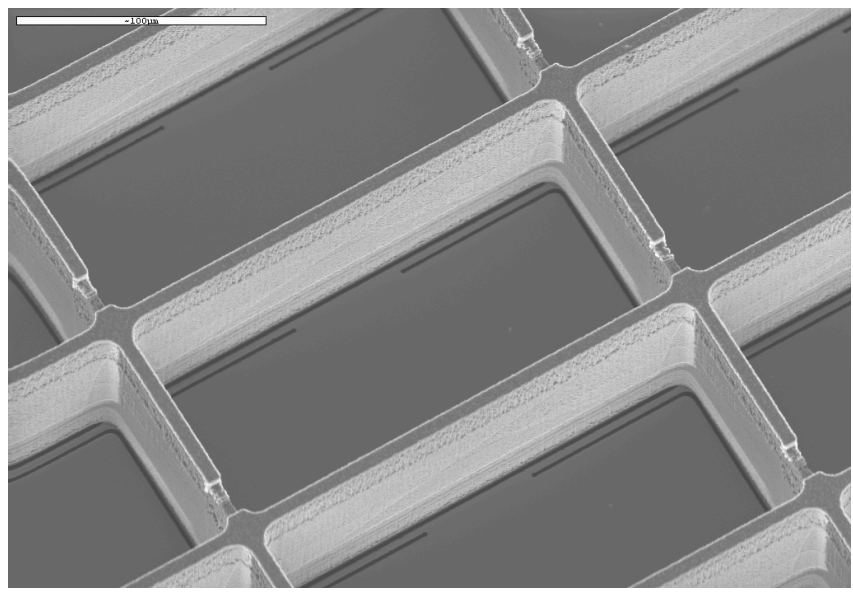

(b)

Figure 2. Secondary electron micrographs of a $128 \times 64$ MSA die (scale indicates $100 \mu \mathrm{m}$ ). (a) Front-side view shown without light shields for clarity. (b) Back-side view.

alignment inspections, (b) electrical continuity and isolation tests, (c) shutter release verification, (d) cryogenic magnetic property characterization, (e) cryogenic shutter bowing characterization, and (f) shutter in-plane twisting resistance tests. Tests performed on fully processed, but unpackaged 128 x 64 MSA dice include (g) cryogenic magneto-mechanical stiffness characterization, and (h) accelerated cryogenic lifetests. Tests performed on fully packaged (i.e. silicon substrate and PCB) 128 x 64 MSA include (i) electrical latching and two dimensional addressing tests, (j) optical functionality tests, (k) accelerated cryogenic lifetests, (l) thermal cycling tests, $(\mathrm{m})$ vibration and acoustical tests, and (n) radiation exposure tests.

\section{MAGNETIC ROTISSERIE LIFETEST METHOD}

One important component of the MSA test plan is accelerated cryogenic lifetesting of unpackaged $128 \times 64 \mathrm{MSA}$ dice. The goal of this test is to actuate shutters to $10^{6}$ actuations, one order of magnitude beyond the $100 \%$ mission lifetime requirement of $10^{5}$ actuations, in a cryogenic environment and evaluate the resultant failures. In the MSA flight concept, shutter actuation is generated magnetically through the interaction between an external magnetic field provided by a permanent tripole magnet and the cobalt-iron film deposited onto each shutter blade. The specific nature of this 
interaction is dependent upon the tripole magnet geometry. A tripole magnet has been modeled and constructed such that the magnetic field lines, emanating and terminating from the three poles, assume a carefully prescribed shape as illustrated in Figure 3. As the tripole magnet is scanned past a shutter cell at a specific distance above the pole tips, the shutter blade is torqued to align with the direction of the applied magnetic field vector. The cobaltiron film on the shutter blade initially magnetizes in the applied field direction and then rotates to follow the rotating magnetic field vector until it is fully open against the back-side electrode on the silicon frame side-wall at which time it can be electrostatically latched open.

Though the scanning tripole magnet actuation method with electrostatic latching has been successfully demonstrated on packaged and unpackaged $128 \times 64$ MSA at room temperature and $30 \mathrm{~K}$ and is ideal for the flight MSA design [3], the scanning mechanism required to translate the tripole magnet back and forth operates very slowly: one complete sweep takes $\sim 10 \mathrm{~s}$. At this rate, a single lifetest to $10^{6}$ actuations would require $\sim 116$ days to complete; too long given the aggressive MSA development schedule. In order to acquire lifetest data to refine the fabrication process in a timely manner, accelerated lifetesting is necessary.

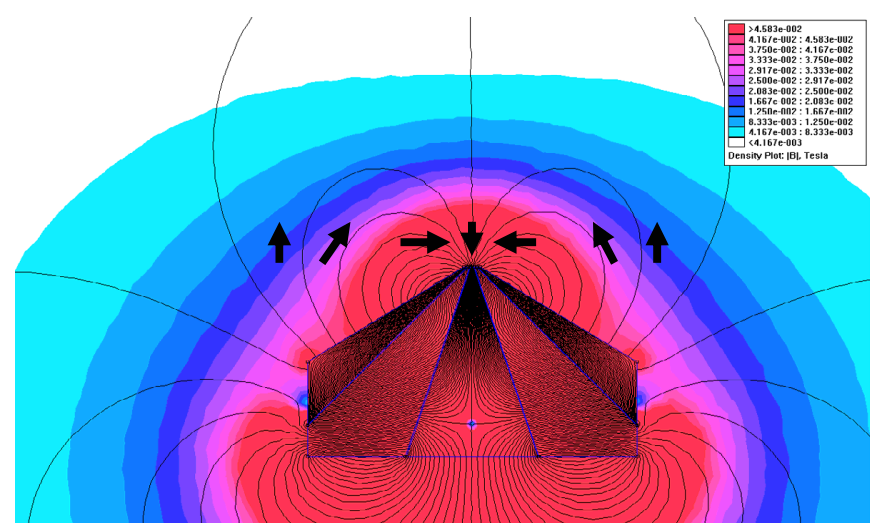

Figure 3. Flux model of tripole magnetic field. Black arrows indicate the rotation of the magnetic field vector at different locations above the tripole magnet tip.

To accomplish accelerated lifetesting, a rapid magnetic actuation method, called the magnetic rotisserie method, was developed. Instead of slowly translating a tripole magnet, the magnetic rotisserie rapidly spins a MSA within a stationary, unidirectional, homogenous magnetic field to simulate the rotating magnetic field vector required to open the shutters. The shutter actuation sequence (top view) is illustrated in the schematic diagram of Figure 4 (not drawn to scale). The green arrows indicate the direction of the homogeneous magnetic field generated between two electromagnet poles (green rectangles). The two black lines and black dot represent, in cross-section, the silicon frame support structure and torsion flexure, respectively, of a single shutter cell. The blue and red arrows represent a shutter blade: the color and arrow direction indicate the magnetization direction of the cobalt-iron magnetic material. The actuation sequence begins at $0^{\circ}$ with the shutter cell oriented such that the shutter blade is parallel to the homogeneous field direction. The homogenous field, $\mathrm{H}$, is turned on and the cobalt-iron magnetic material is initially magnetized away from the torsion flexure as indicated by the blue arrow. As the shutter cell is spun in a clockwise direction (indicated by the orange arrow), a torque is produced in the magnetized cobalt-iron material which attempts to maintain alignment with the applied homogeneous magnetic field by rotating about the torsion flexure axis against the restoring spring force of the torsion flexure as indicated at $45^{\circ}$. At $90^{\circ}$, the shutter blade makes first contact with the silicon frame support structure: the shutter cell is fully open. As the shutter cell continues to spin, the shutter is pushed along by the silicon frame support structure: the shutter cell is still fully open. At $\sim 180^{\circ}$, the combined effects of the torsion flexure restoring force and a sudden magnetic reversal of the cobalt-iron magnetization direction, now pointing towards the torsion flexure, causes the shutter blade to swing closed as indicated by the red arrow. As the shutter cell continues to spin, the remagnetized cobalt-iron again experiences a torque which forces the shutter blade to rotate open $\left(225^{\circ}\right)$ and the entire actuation process repeats itself: $270^{\circ}$ fully open, $315^{\circ}$ fully open against silicon frame support. At $360^{\circ}$, the combined effects of the torsion flexure restoring force and another magnetic reversal in the cobalt-iron again causes the shutter blade to swing close as indicated by the blue arrow. Thus, one revolution of the shutter cell in the magnetic rotisserie results in two complete shutter actuations.

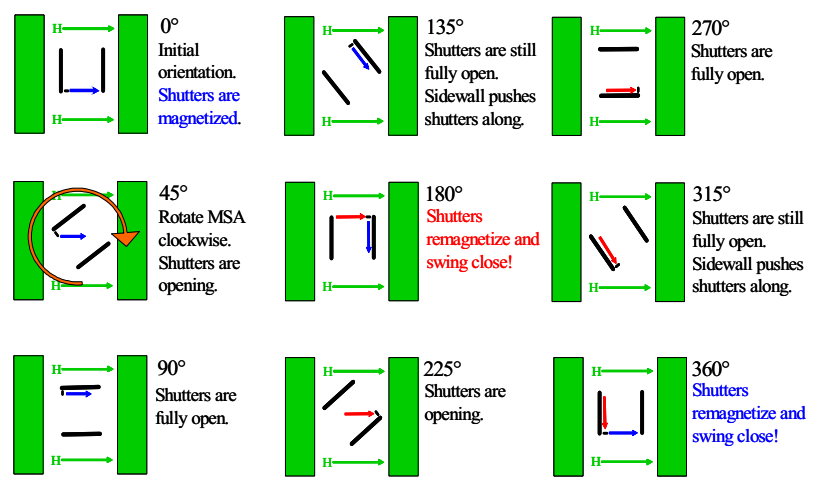

Figure 4. Schematic diagram (top view) illustrating the magnetic rotisserie actuation sequence.

Two accelerated cryogenic lifetest facilities have been constructed based upon the magnetic rotisserie method: a liquid nitrogen-cooled system $\left(\mathrm{LN}_{2}\right.$ rotisserie) and a liquid helium-cooled system (LHe rotisserie). The LHe rotisserie is depicted in Figure 5. Unpackaged MSA dice samples are inserted into a nonmagnetic, liquid helium dewar and suspended above the surface of the liquid helium coolant. The dewar is situated within a uniform, homogeneous magnetic field generated by a pair of water-cooled electromagnets. The magnetic field strength is adjustable $(-3 \mathrm{~T} \leq$ $\mathrm{H} \leq 3 \mathrm{~T})$ and monitored by a Gaussmeter. The MSA samples are attached to a drive shaft and motor assembly which provides the spinning motion which can attain shutter actuation rates up to 30 Hz. A mechanical counter monitors drive shaft revolutions which are converted to total shutter actuations. The sample temperature is monitored by two silicon diode sensors mounted on the MSA sample holder. The $\mathrm{LN}_{2}$ rotisserie and the LHe rotisserie are capable of cryogenic testing between $90 \mathrm{~K} \leq \mathrm{T} \leq 105 \mathrm{~K}$ and $8 \mathrm{~K} \leq$ $\mathrm{T} \leq 30 \mathrm{~K}$, respectively.

MSA samples are optically inspected through a set of dewar windows via transmitted light microscopy. Figure 6 displays typical images of an entire $128 \times 64$ MSA without light shields in the closed $\left(0^{\circ}\right)$ and open $\left(345^{\circ}\right)$ positions that demonstrate the validity of the magnetic rotisserie method for actuating shutters to 
the open position ${ }^{\mathrm{e}}$. Use of fast camera shutter speeds during image acquisition also verifies shutter actuation while the MSA sample is spun at $900 \mathrm{rpm}$ (or $30 \mathrm{~Hz}$ shutter actuation rate).

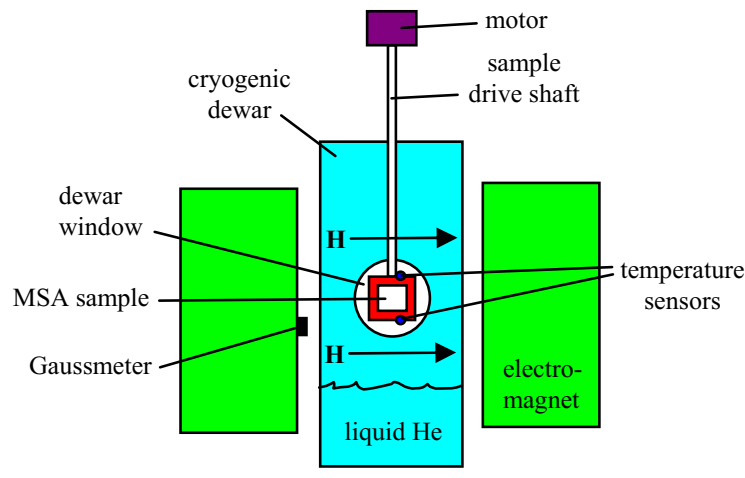

Figure 5. Schematic diagram (side view) of cryogenic magnetic rotisserie.
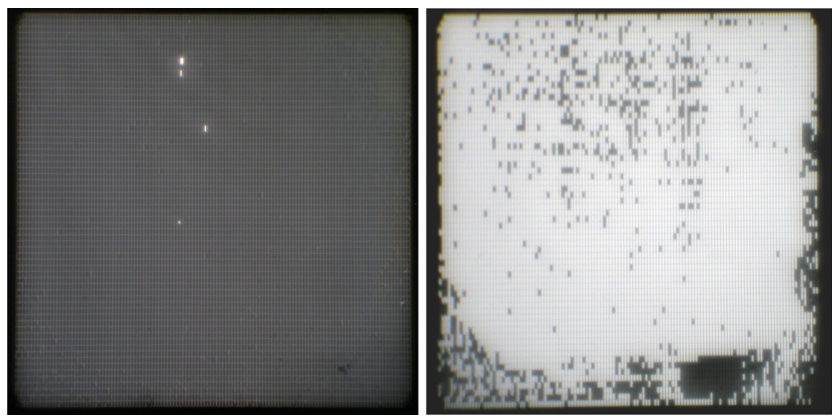

Figure 6. Transmitted light micrographs of a $128 \times 64$ MSA without light shields in the LHe rotisserie in the (a) fully closed position at $0^{\circ}$ and, (b) fully open position at $180^{\circ}$ with $H=0.4 \mathrm{~T}$. The active area of the MSA is $1.3 \mathrm{~cm}$ across.

Accelerated lifetests were conducted on five MSA samples with the magnetic rotisserie method to evaluate two failure conditions for individual shutter cells: "failed open" (light leaking through a supposedly closed shutter cell) and "failed closed" (light blocked by a supposedly open shutter cell). The as-received, beginning-of-life (BOL), condition of all five MSA samples were imperfect, containing $\leq 3 \%$ "failed open" and $\leq 11 \%$ "failed closed" failures. Shutter cells exhibiting these BOL failures were excluded from subsequent lifetest analyses. On average, over 7,500 shutter cells per sample participated in cryogenic lifetesting.

None of the rotisserie-tested MSA samples contained light shields (LS). The cobalt-iron on each shutter of all of the MSA samples was of an earlier design, configured as a single square or rectangular pad instead of multiple stripes. One sample, a $128 \mathrm{x}$ 128 MSA from an earlier design with a $100 \times 100 \mu \mathrm{m}$ shutter cell size (SOI 27-1), was lifetested at room temperature in ambient air. Cryogenic lifetesting between $90-105 \mathrm{~K}$ in nitrogen vapor was performed on four $128 \times 64$ MSA samples of a more recent design with a $100 \times 200 \mu \mathrm{m}$ shutter cell size (SOI 47-C, SOI 48-C, SOI 61-B, SOI 61-D). Two samples (SOI 47-C, SOI 61-B) underwent additional cryogenic lifetesting between $8-30 \mathrm{~K}$ in helium vapor.

\footnotetext{
e This particular MSA sample had known processing imperfections (i.e.: missing and stuck shutters) but was still suitable for verifying shutter actuation.
}

The torsion flexure geometry (width and length) of all of the MSAs varied between the samples.

Images of the entire MSA in an open and closed position were recorded at $\mathrm{BOL}$ and repeated at a minimum at every order of magnitude of shutter actuation throughout the lifetest. The total change in the number of "failed open" and "failed closed" shutters from one inspection to the next was counted and the associated number of shutter actuations was noted. A summary of the lifetest results is presented in Figure 7.

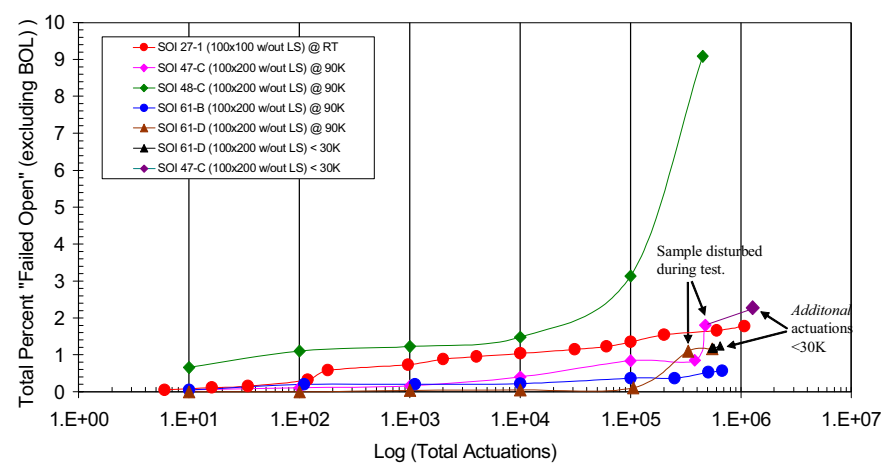

(a)

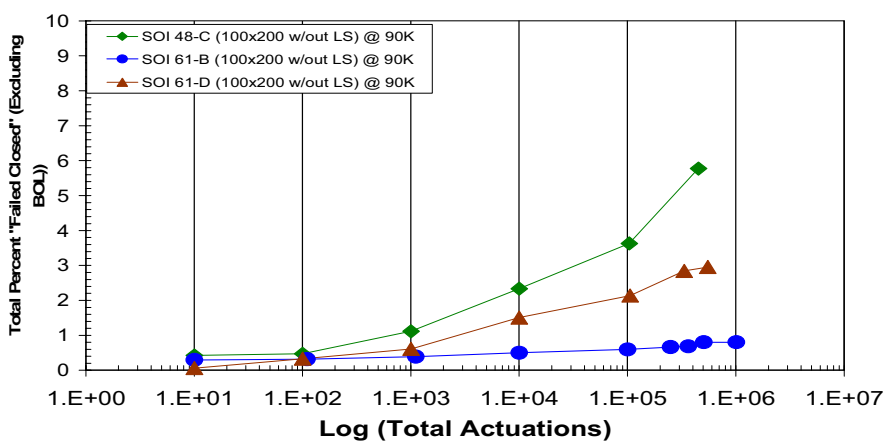

(b)

Figure 7. Summary of lifetest results for (a) "failed open" failures, and (b) "failed closed" failures.

For four of the MSA samples lifetested the "failed open" failure rate increased logarithmically adding $<2.3 \%$ failures at $10^{6}$ actuations. Scanning electron microscopy (SEM) analysis indicates that the predominant failure mode in these cases was shutters missing due to fracture on the torsion flexure or near the shutter blade neck. The anomalous behavior of the "failed open" failures of sample SOI 48-C is currently under investigation. The "failed closed" failure rates also increased with actuations. The primary failure mode was contact between the shutter blades against the silicon frame side-walls preventing shutter actuation. This type of failure would occasionally "heal" itself: a shutter cell qualifying as a "failed closed" failure in one optical inspection would not always qualify as a failure in the next inspection. Slight misalignments between the shutter blade and the surrounding silicon frame and out-of-plane shutter blade twisting due to low cobalt-iron coercivity may be the cause of the "failed closed" failures. Improving the front-to-back alignment with newly acquired fabrication tools and modifying the cobalt-iron magnetic pad into a multiple stripe design with higher coercivity may reduce the number of "failed closed" shutters in future accelerated lifetests. 


\section{MAGNETO-MECHANICAL STIFFNESS}

Since the functional performance of shutter actuation is dependent upon a combination of effects, namely the restoring spring force of the torsion flexure and the magnetic reversal in the cobalt-iron material, the magneto-mechanical stiffness is an important parameter for MSA design purposes. The magnetic rotisserie lifetest method conveniently enables this measurement for all shutters in a MSA in parallel and at cryogenic temperatures.

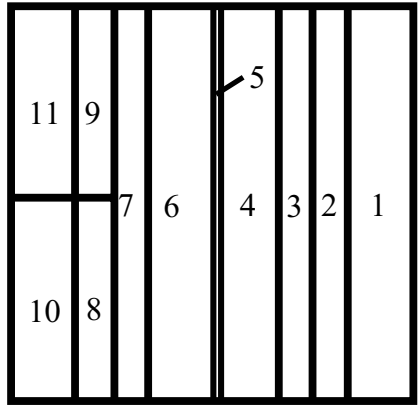

(a)

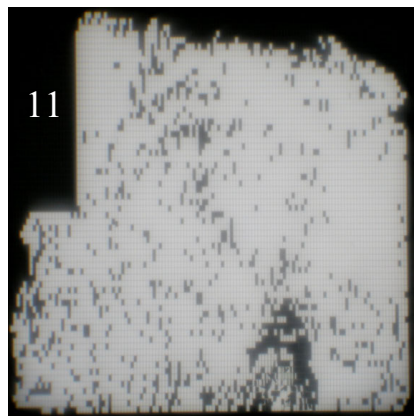

(c)

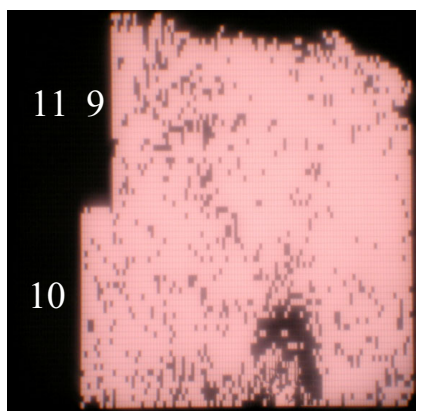

(e)

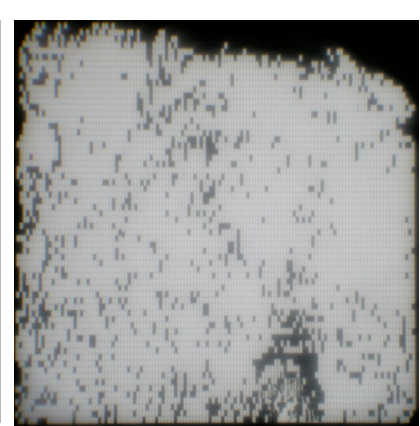

(b)

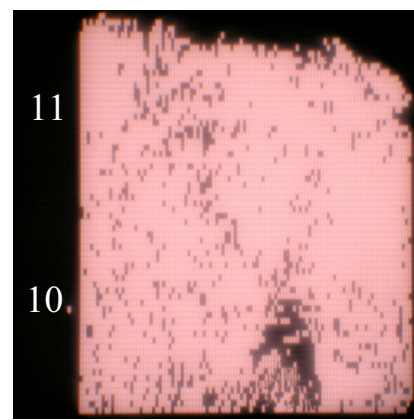

(d)

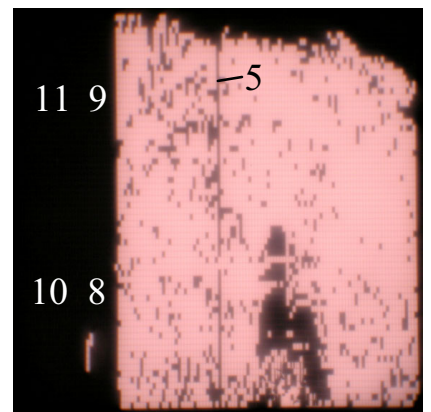

(f)
Figure 8. Magneto-mechanical stiffness characterization of "pathfinder" $128 \times 64 \mathrm{MSA}$. (a) Schematic of 11 different test regions. (b) All regions open at $0.200 \mathrm{~T}$. (c) Region 11 closed at $0.160 \mathrm{~T}$. (d) Region 10 closed at $0.145 \mathrm{~T}$. (e) Region 9 closed at $0.130 \mathrm{~T}$. (f) Region 8 closed (95\%), Region 5 closed (95\%) at $0.120 \mathrm{~T}$.

A "pathfinder" 128 x 64 MSA sample was fabricated containing 11 test regions each with a different torsion flexure geometry (variables were width and length) and/or magnetic stripe design (variables were number of stripes per shutter and stripe width) and is schematically represented in Figure 8 (a). To characterize the magneto-mechanical stiffness, the "pathfinder" MSA sample was placed in the magnetic rotisserie lifetest apparatus and slowly spun in the magnetic field $(\mathrm{H}=0.200 \mathrm{~T})$ until all shutters in the array were open. The sample was held at this angle (relative to the applied magnetic field direction) and the magnetic field strength was slowly decreased. As the torsion flexure restoring force and magnetic reversal in the cobalt-iron of a particular test region combined to overcome the applied magnetic field, the shutters within that test region swung close. Shutters within test regions that were magneto-mechanically stiff closed at higher applied magnetic fields than shutters within test regions that were magneto-mechanically more compliant as illustrated in Figure 8 (b-f). These results have been used to finalize a specific torsion flexure geometry and magnetic stripe design for the next generation of MSA.

\section{CONCLUSIONS}

The MSA development team at the NASA GSFC has successfully fabricated $128 \times 64$ MSA devices. A comprehensive test plan has been implemented and MSA-specific cryogenic testing and characterization techniques have been developed. The magnetic rotisserie lifetest method which has been demonstrated at cryogenic temperatures indicate a logarithmic "failed open" failure rate that adds $<2.3 \%$ failures at $10^{6}$ actuations. This method has increased our understanding of different failure modes and provides a means to predict device reliability. Near-term efforts include additional cryogenic lifetesting on the next generation 128 x 64 MSA samples with light shields and fabrication of flight-like 384 x 175 MSA devices.

\section{REFERENCES}

1. H.S. Stockman, The Next Generation Space Telescope: visiting a time when galaxies were young, The Association of Universities for Research in Astronomy, Inc., 1997.

2. S.H. Moseley, et al., "Status of the Development of $128 \times 128$ Microshutter Array", in MOEMS and Miniaturized Systems, Proceedings of SPIE 4178, 2000.

3. A.S. Kutyrev, et al., "Programmable microshutter arrays for the JWST NIRSpec", accepted for publication in the Journal on Selected Topics in Quantum Electronics on Optical Microsystems, March 2004.

4. M.J. Li, et al., "Fabrication of Microshutter Arrays for Space Application", in Microelectronic and MEMS Technologies, Proceedings of SPIE 4407, 2001.

5. D.B. Mott, et al., "Magnetically Actuated Microshutter Arrays", in Micro-machining and Microfabrication, Proceeding of SPIE 4561, 2001.

6. M.J. Li, et al., "Microshutter Arrays for Near-Infrared Applications on the James Webb Space Telescope", Proceedings of SPIE 4981, 2003. 